El Guiniguada • Revista de investigaciones y experiencias en Ciencias de la Educación

\title{
ANÁlisis SOBRE LA INFLUENCIA DE LAS ESTRATEGIAS ORALES \\ PREVIAS AL DESARROLLO DE ACTIVIDADES DE COMPRENSIÓN AUDITIVA
}

\section{ANALYSIS ON THE INFLUENCE OF ORAL STRATEGIES PRIOR TO THE DEVELOPMENT OF LISTENING COMPREHENSION ACTIVITIES}

\author{
Zeus Plasencia Carballo \\ Natalia Pérez Santana \\ CEIPS Chamberí (Santa Cruz de Tenerife) / \\ Investigadora Universidad de La Laguna
}

Recibido: 25/01/2015/Aceptado: 14/04/2015

\section{RESUMEN}

De entre las siete destrezas que promueven la adquisición de una lengua, en el presente estudio nos hemos centrado exclusivamente en la relacionada con la comprensión auditiva, dado que es una de las que mayor dificultad plantea durante el aprendizaje de una lengua extranjera y, por lo general, la destreza que menos atención recibe dentro de las aulas de Lengua Extranjera.

Con vistas a identificar qué aspectos se consideran más relevantes durante el desarrollo de la comprensión auditiva, se ha llevado a cabo una investigación en un aula de Educación Secundaria Obligatoria (ESO), limitando el alcance del estudio a las influencias que puedan ejercer las actividades orales previas al desarrollo de una actividad de comprensión auditiva (pre-listening, listening y post-listening). La experiencia en el aula y los datos obtenidos evidencian un resultado positivo al trabajar los conocimientos previos, a través de estrategias orales antes de la realización de actividades de comprensión auditiva.

Palabras clave: conocimientos previos, destrezas, comprensión auditiva, estrategias orales, lengua extranjera. 


\title{
Zeus Plasencia Carballo y Natalia Pérez Santana
}

Análisis sobre la influencia de las estrategias orales previas al desarrollo de actividades de comprensión auditiva

\begin{abstract}
Among the seven skills that promote the acquisition of a language, we have focused on the listening comprehension, due to the difficulty it entails when learning a second language and, generally, the least worked skill in Foreign Languages lessons.

With a view to identify the most relevant aspects during the development of listening comprehension activities, this investigation has been carried out in a Compulsory Secondary Education class, fencing its approach to the influences that previous oral activities can imply during the development of listening comprehension activities (pre-listening, listening and post-listening). The experience and the data obtained during the investigation give a clear evidence of positive results regarding the influence of oral strategies prior to the development of listening comprehension activities.
\end{abstract}

Keywords: previous knowledge, skills, listening comprehension, oral strategies, foreign language.

\section{INTRODUCCIÓN}

En la actualidad, es notable la importancia que presentan los conocimientos previos en la mejora de las destrezas de un idioma. De hecho, esta realidad se acentúa en la enseñanza de una lengua extranjera, como puede ser el inglés. De entre las siete destrezas que promueven la adquisición de una lengua, para el presente estudio nos hemos centrado exclusivamente en la relacionada con la comprensión auditiva, dado que esta es una de las que mayor dificultad plantea durante el aprendizaje de una lengua extranjera y, por lo general, la destreza que menos atención recibe dentro de las aulas de Lengua Extranjera ${ }^{1}$.

Estudios recientes, como los realizados por Chang (Chang, 2012), corroboran que las estrategias previas al desarrollo de actividades de comprensión auditiva favorecen el entendimiento y la superación de dichas actividades.

La intencionalidad del presente artículo es compartir las experiencias y resultados obtenidos durante una investigación llevada a cabo durante el curso 2012-2013 en un aula de $2^{\circ}$ de Educación Secundaria Obligatoria (ESO), limitando el alcance del estudio a las influencias que puedan ejercer las actividades orales previas al desarrollo de una actividad de comprensión auditiva en grupos de la ESO.

1 Véanse resultados obtenidos en las Pruebas de Diagnóstico 2011-2012 del Instituto Canario de Evaluación y Calidad Educativa (ICEC). 


\section{Zeus Plasencia Carballo y Natalia Pérez Santana}

Análisis sobre la influencia de las estrategias orales previas al desarrollo de actividades de comprensión auditiva

Atendiendo a todo lo anterior, el diseño y la puesta en práctica de estrategias orales que faciliten la comprensión auditiva dentro de un aula de secundaria se convertirá en el objetivo principal de discusión a través de las siguientes páginas; además de analizar las ventajas que puedan proporcionar las estrategias orales.

A pesar de un sensible cambio metodológico, un equipo docente mejor preparado y la introducción de las Tecnologías de la Información y Comunicación (TIC), en las aulas de Lengua Extranjera se sigue abordando la comprensión auditiva de manera somera y mecánica: a través de un mínimo de dos escuchas, por lo general, y con la realización de tareas de comprensión sobre los contenidos de dicha escucha como cierre del ejercicio de comprensión auditiva. Parece que existiese una metodología restrictiva en cuanto a la manera de plantear el enfoque de las comprensiones auditivas; sin embargo, tanto los currículos educativos de las Comunidades Autónomas (CCAA), como el Marco Común de Referencia Europeo para las Lenguas (MCREL) conceden carta blanca sobre el contenido que los estudiantes escucharán (input), cómo se les preparará para dicho ejercicio o qué se les exigirá.

Citando el Bloque I del currículo de Lengua Extranjera de la CCAA de Canarias, podemos observar que en el punto tercero del apartado escuchar se hace una mención especial al desarrollo y uso de estrategias que impulsen la comprensión oral: "uso del contexto verbal y no verbal y de los conocimientos previos sobre el tema transferidos desde las lenguas conocidas a la lengua extranjera, identificación de vocablos internacionales y palabras claves [...]". Para nuestra investigación tomamos como base el citado punto, entre otros, dando especial protagonismo a los mencionados conocimientos previos.

En su momento, Piaget argumentó que las experiencias y los conocimientos ya interiorizados por cualquier persona son la base para asentar nuevos aprendizajes (Piaget, 2009). Partiendo de este pilar constructivista, descubrimos hoy escuelas e institutos donde el profesor se convierte en un facilitador del conocimiento, quien actuará como guía durante el proceso de construcción de conocimientos por parte del alumno y quien, además, creará las condiciones óptimas para que se dé lugar a dicho proceso. Por ello, en la actualidad, dentro de las aulas se impulsa el conocido como aprendizaje significativo, donde el alumno descubre y construye el conocimiento, partiendo del diálogo y la interacción conjunta. En palabras de Coll, "un aprendizaje es tanto más significativo cuantas más relaciones con sentido es capaz de establecer el alumno entre lo que ya conoce, sus conocimientos previos y el nuevo contenido que se le presenta como objeto de aprendizaje" (Coll, 1990:50). En otras palabras, un alumno aprende un contenido cuando es capaz de atribuirle un significado, y ello se produce cuando se entablan similitudes y conexiones con lo que este ya conoce, es decir, sus conocimientos previos como se especifica en el anteriormente mencionado punto tercero del bloque I del currículo de Secundaria. 


\section{Zeus Plasencia Carballo y Natalia Pérez Santana}

Análisis sobre la influencia de las estrategias orales previas al desarrollo de actividades de comprensión auditiva

Y ¿de dónde parten los conocimientos previos de los alumnos? ¿Cómo los relacionan con la L2 (lengua extranjera)? Si continuamos por la línea del contexto más próximo al alumno, la propia lengua materna (L1) es la respuesta a la primera cuestión; mientras que para la segunda, varios son los agentes implicados -el propio alumno, el factor motivación, las facilidades o dificultades para aprender una lengua extranjera, el propio profesor, etc.-. Sin embargo, uno de los más determinantes es la contextualización. Recordemos, pues, que cualquier conocimiento nuevo que vayamos a aprender, si está en consonancia con nuestro contexto más cercano, será mucho más fácil de asimilar. Este último aspecto cobra bastante importancia dentro de nuestro estudio, puesto que, durante una comprensión auditiva, si el alumnado conecta con el contenido del audio, debido a que este ofrece algún aspecto o particularidad que capte su atención, el interés que se genere será directamente proporcional al asentamiento de nuevas nociones, conocimientos y se producirá el fenómeno del aprendizaje. Por otro lado, el profesor, en su papel de facilitador, deberá realizar las conexiones oportunas entre el texto oral y el contexto particular del alumno.

\section{Metodología}

Como ya hemos puesto de manifiesto anteriormente, nuestro análisis fue llevado a cabo en un curso de $2^{\circ}$ de $\mathrm{ESO}^{2}$, en un instituto público canario donde el alumnado con el que se trabajó pertenecía a un grupo socio-económico medio-bajo. Es importante puntualizar este último dato, ya que nos mueve estudiar los resultados obtenidos en grupos que presenten mayor dificultad, y no con grupos que, por regla general, reciben ayudas o clases extraescolares que promueven su aprendizaje de la lengua extranjera fuera del ámbito escolar.

Durante nuestra investigación, y atendiendo a la necesidad imperante de que existiese una relación desde el primer momento entre el alumnado y el audio que iban a escuchar, se ofreció una serie de patrones o pautas similares a las que se deberían llevar a cabo durante la comprensión escrita, es decir, se establecieron tres fases: antes, durante y después de la escucha, siguiendo la línea de Rost ${ }^{3}$ (Rost, 2002). Estas pautas, y otras consideraciones pertinentes, también aparecen reflejadas en el MCERL, en el apartado 7.3.2.2. A continuación, desglosamos los mencionados tres momentos de la comprensión auditiva y procedemos a su descripción:

2 Tomando como referencia el mismo nivel educativo analizado durante las últimas pruebas diagnóstico 2012 en Canarias.

3 Según Rost, la información nueva interactúa con los conocimientos previos para confirma o actualizar dicha información, puesto que tanto el entendimiento como el no entendimiento impulsa a inferir y/o razonar, produciendo así nuevos recuerdos o aprendizajes en el individuo. 


\section{Zeus Plasencia Carballo y Natalia Pérez Santana}

Análisis sobre la influencia de las estrategias orales previas al desarrollo de actividades de comprensión auditiva

1. Antes de LA ESCUCHA

a) Presentación/Introducción.- Se informa al alumno de que escuchará un audio y trabajará sobre el mismo.

b) Se hablará sobre el título del audio, sobre qué puede tratar, se mostrará imágenes o material audiovisual de apoyo en consonancia con el tema y se tratará de conectarlo con el contexto inmediato del alumno.

2. Durante la escucha

a) Escucha(s).- Se procederá a escuchar el audio tantas veces como se crea conveniente.

b) Asimilación.- Se trabajará en i) parejas, ii) pequeño grupo y iii) toda la clase, sobre aspectos concretos que dirijan al entendimiento del audio. El audio podrá seguir utilizándose como apoyo durante este punto.

\section{Después de la escucha}

a) Expansión.- Los métodos empleados para esta tarea se pueden extrapolar a futuras tareas de comprensión auditiva.

b) La costumbre en este tipo de prácticas conllevará una mayor agilidad por pare del alumnado, quien desarrollará y mejorará las estrategias para la comprensión auditiva, aumentando así su capacidad de comprensión y la complejidad de los futuros textos orales.

Por otro lado, el MCERL también contempla estrategias de comprensión en su punto 4.4.2.4, los cuales tomamos en consideración durante nuestra investigación en el aula. Dichas estrategias son:

1. Planificación.- Identificación de contexto y conocimientos. Puesta en marcha de esquemas apropiados.

2. ENCUADRE.- Expectativas respecto a la organización y contenido de lo que va a ocurrir.

3. INFERENCIA.- Llenado de vacíos aparentes en el mensaje.

4. Comprobación DE Hipótesis.- Manera en la que se interpreta la situación.

El estudio en el aula se plantea a través del diseño de una unidad didáctica cuyo centro temático es la música y el baile. Partiendo de dicha base, se hizo uso de los audios incluidos en el manual del alumno y de textos que se adaptaron para nuestro propósito. De igual manera, planteamos pautas de pre-listening, listening y post-listening en cada una de las actividades de comprensión oral. Dichas actividades se describen, brevemente, a continuación: 


\section{Zeus Plasencia Carballo y Natalia Pérez Santana}

Análisis sobre la influencia de las estrategias orales previas al desarrollo de actividades de comprensión auditiva

a) Capoeira and Street dance classes.- Audio-texto basado en dos pósters informativos sobre clases de Capoeira y Street dance.

b) Music for Pets.- Audio-texto basado en una investigación sobre los gustos musicales de las mascotas.

c) Going to a concert.- Audio basado en la invitación a un concierto entre dos amigos.

A continuación, procederemos a la descripción de las fases en cada una de las comprensiones auditivas mencionadas anteriormente.

En la fase de pre-listening del audio-texto A, procedemos en primer lugar a contextualizar. Se escribe en la pizarra las palabras "Capoeira" y "Street dance" y se pregunta a los alumnos si conocen dichas palabras y si podrían explicar qué son o significan. También, se pregunta si los alumnos practican alguno de esos tipos de bailes, si les gustan, si conocen, etc. Tratamos de conectar el audio con su entorno más cercano. En segundo lugar, creamos expectativas y dirigimos el interés a través de la proyección de dos vídeos cortos sobre los bailes de la Capoeira y el Street dance. La clase, conjuntamente, relaciona las palabras escritas en la pizarra con los vídeos mostrados. En tercer lugar, verificamos que los alumnos saben sobre qué tema vamos a enfocar la actividad. Lo constatamos a través de preguntas al grupo en general o individuales.

En la siguiente fase, listening, ejecutaremos el audio una primera vez con el fin de que los alumnos se familiaricen con la grabación (duración, acento, rapidez, vocabulario familiar, vocabulario nuevo, etc.). Una segunda escucha propiciará que el alumnado reconozca y perciba más palabras y detalles que en la primera escucha. Así, comenzarán a inferir significados y a generar hipótesis a cerca del audio. A continuación, y tras una tercera ejecución, comprobaremos qué han entendido los alumnos a cerca del audio a través de preguntas individuales. Más tarde, hacemos una puesta común sobre las conjeturas obtenidas por el grupo. En este punto, el profesor orientará siempre, no se limitará a decir si es correcta o no la conclusión del alumno. Para finalizar con este nivel, ejecutamos por cuarta vez, y última en esta etapa, el audio para verificar la hipótesis grupal. Es decir, comprobamos en la última escucha que los comentarios ofrecidos por los alumnos son certeros y coinciden con lo escuchado.

Durante el post-listening, acompañaremos el audio con la lectura del mismo. De esta manera, al leer la transcripción del audio, el alumno podrá trabajar sobre el vocabulario nuevo o detalles inesperados que no pudieron asimilar durante las escuchas. Además, de esta manera se relaciona el factor fonético con la grafía del término. Finalmente, y para concluir con la actividad de comprensión auditiva, se realizaron los ejercicios propuestos en el manual para el audio trabajado. Así, en última instancia, comprobaremos la efectividad de la comprensión auditiva por parte del alumnado. 


\section{Zeus Plasencia Carballo y Natalia Pérez Santana}

Análisis sobre la influencia de las estrategias orales previas al desarrollo de actividades de comprensión auditiva

Tras esta primera actividad de comprensión auditiva, advertimos que el alumnado no es consciente de que el ejercicio que van a realizar tendrá que ver con una comprensión auditiva, por lo que el alumno no sabe con exactitud qué se va a hacer en clase hasta que se comienza a ejecutar las escuchas. Por un lado, es importante destacar que las actividades orales se manifiestan, sobre todo, en la fase de pre-listening y son dirigidas por el profesor principalmente. Es decir, el profesor mantiene diálogos con el alumnado; sin embargo, entre alumnos no interactúan. Por otro lado, cabe comentar que el número de escuchas se repite hasta cuatro veces. Este tipo de repeticiones sería un tanto tedioso para el profesor como para el alumno, si el audio fuera muy largo. Por último, es reseñable que la escucha del audio acompañada de la lectura ayuda bastante en la asimilación de conceptos e ideas; además de familiarizar sonidos y grafías.

En el audio-texto B, llevamos a cabo la contextualización en la primera fase, prelistening, donde escribimos en la pizarra las palabras "Pets" y "Music", como ya hicimos con el primer audio, y preguntamos a los alumnos qué tipo de conexión creen que pueda existir entre ambas palabras. A continuación, creamos expectativas y dirigimos el interés de los alumnos con el fin de entablar posibles conexiones con el contexto inmediato del alumnado y con el audio, por lo que se les pregunta si tienen mascotas y si han comprobado algún tipo de comportamiento o reacción en cuanto a la música que escuchan. En definitiva, creamos expectativas e inquietudes por las preguntas que se formulan. Seguidamente, activamos algo de vocabulario específico sobre el tema: tipos de músicas, nombres de mascotas, etc.; a través de un diálogo entre el profesor y los alumnos.

Durante la fase de listening, ejecutamos el audio por primera vez para familiarizar al alumnado con las características que este presente (duración, acento, rapidez, vocabulario familiar, etc.), como se hizo con la actividad de comprensión auditiva A. Aprovechamos este paso para preguntar a los alumnos sobre sus primeras impresiones respecto al audio: ¿Sobre qué trata el audio? ¿Has identificado algún personaje? ¿Reconoces algo del vocabulario? Escribimos sus aportaciones en la pizarra con el fin de orientar el audio y rescatar vocabulario básico de este. Con la segunda escucha, se pretende que el alumnado comience a reconocer más palabras y percibir más detalles que la primera vez. Estos comienzan a inferir significados y a generar hipótesis. Preguntamos sobre nuevos aspectos o detalles que hayan podido reconocer y qué sentido va adquiriendo el audio para ellos. También, hacemos preguntas más específicas sobre el audio, comprobando que los alumnos no hayan perdido el sentido del mismo, aunque no lo entiendan en su totalidad. La tercera escucha viene de la mano con la lectura de la transcripción del audio, haciendo preguntas más complejas que puedan resolver también a través del texto. Este acompañamiento de la lectura con el audio promueve el aprendizaje de la pronunciación y énfasis adecuado de algunas palabras. Finalmente, en el apartado de post-listening, creamos las 


\section{Zeus Plasencia Carballo y Natalia Pérez Santana}

Análisis sobre la influencia de las estrategias orales previas al desarrollo de actividades de comprensión auditiva

condiciones adecuadas para promover un debate en el cual pueda participar todo el grupo sobre las impresiones obtenidas del audio. El profesor, esta vez, participa pero está más interesado en las opiniones que pueda ofrecer el grupo. También, como en la actividad A, se proponen los ejercicios de consolidación facilitados por el manual, donde se comprueba, en última instancia, la efectividad de la comprensión auditiva por parte del alumnado.

Durante la puesta en práctica de esta actividad, los alumnos son advertidos de la escucha a mitad del proceso. También, se reduce el número de escuchas a tres, siendo más concretos a la hora de generar preguntas relacionadas con el audio y dirigiendo el interés hacia este. A diferencia del ejercicio A, el profesor interactúa con los alumnos y promueve que el debate se mantenga entre los propios alumnos. Sin embargo, no se enfatiza el trabajo en pequeño grupo. Por último, advertimos que durante el pre-listening, sólo existe la asistencia oral y contextual basada en las experiencias de los propios alumnos. Es decir, no existe ayuda audio visual (vídeos) como en otras ocasiones.

En lo referente al audio C, Going to a concert, en primer lugar se procede a la contextualización, poniendo en preaviso al alumnado sobre la actividad de listening que se va a desarrollar. Escribimos en la pizarra la palabra "Concert" y procedemos a conectar con el contexto inmediato del alumnado, activando conocimientos previos y vocabulario relacionados con el centro temático en el que se trabajará: stage, spotlight, etc. A continuación, se invita a los alumnos a trabajar en pequeño grupo durante varios minutos para que puedan crear hipótesis sobre qué tipo de audio escucharán. Su punto de partida para la discusión será la palabra "Concert", anteriormente escrita en la pizarra y todo aquello que se haya comentado en torno a dicha palabra. De este modo, favorecemos la creación de expectativas y dirigimos el interés de los alumnos. Seguidamente, preguntamos a los alumnos por las relaciones que han establecido y las hipótesis resultantes. Así, verificamos si se acerca o no al tema que trata el audio. Promovemos unos minutos más esta interacción entre alumnos escribiendo la palabra "invitation" en la pizarra.

Durante la fase de listening, volvemos a ejecutar una primera vez el audio para que los alumnos se familiaricen con las características del audio; sin embargo, como novedad, dejamos unos minutos para que los alumnos puedan discutir en pequeño grupo qué consideran haber entendido. En la pizarra escribimos las aportaciones de nuestros alumnos y activamos vocabulario conocido e incluimos nuevo. A través de estas aportaciones y del diálogo grupal, los alumnos comienzan a inferir sentidos en el audio. Durante la segunda ejecución del audio, estrechamos el cerco. Es decir, se hacen preguntas más específicas sobre el audio y enfocamos la atención del alumno en aquellos puntos que nos interesen. En pequeño grupo, los alumnos debaten aquello que han entendido durante esta segunda escucha y sobre las preguntas propuestas por el profesor. Finalmente, ejecutamos la grabación una tercera vez con el 


\section{Zeus Plasencia Carballo y Natalia Pérez Santana}

Análisis sobre la influencia de las estrategias orales previas al desarrollo de actividades de comprensión auditiva

fin de comprobar que los alumnos siguen la línea dibujada por el profesor. Realizamos preguntas más específicas, como cuál es el nombre del perro u otros detalles del audio. Siempre, durante esta fase, los alumnos trabajan en pequeño grupo.

Por último, en la etapa de post-listening, promovemos una puesta en común general sobre cuestiones que se han escuchado, lo que han entendido u opiniones obtenidas del audio. Los ejercicios de consolidación serán el punto y final para la actividad, al igual que en otras ocasiones; así, verificamos la efectividad de la comprensión auditiva por parte del alumnado.

En este último caso descrito, observamos que desde el principio se avisó al alumnado sobre el tipo de ejercicio que se iba a realizar. El diálogo no se concentró exclusivamente entre profesor y alumno, sino entre alumnos también. De este modo, se trabajó constantemente en pareja o pequeño grupo; promoviendo que los alumnos se ayuden y resuelvan entre ellos las preguntas que surgían a lo largo de la actividad. Ellos mismos entablan hipótesis, infieren significados y resuelven. También, podemos observar cómo se reactiva vocabulario ya conocido y se introduce nuevo. Esto último se debe, nuevamente, a la interacción constante y amplificada (profesoralumnos, alumnos-alumnos). En última instancia, a lo largo de la actividad se promueve la autonomía de los alumnos constantemente.

\section{Resultados}

Tras haber llevado a cabo el ejercicio de comprensión auditiva, a través de distintos enfoques y empleando distintas estrategias orales, a continuación se facilita un cuadroresumen sobre las estrategias orales empleadas durante las comprensiones orales:

\begin{tabular}{|l|c|c|c|c|c|c|c|c|c|c|c|}
\hline \multirow{2}{*}{ Estrategia oral } & \multicolumn{3}{c}{} & \multicolumn{3}{c|}{ Pre-listening } & \multicolumn{3}{c|}{ Listening } & \multicolumn{3}{c|}{ Post-listening } \\
\hline \multicolumn{2}{|c}{ Audio } & A & B & C & A & B & C & A & B & C \\
\hline Ayuda audio-visual & + & - & - & - & - & - & - & - & - \\
\hline Diálogo (profesor-alumno) & + & + & - & + & + & - & + & + & + \\
\hline Trabajo en pequeño grupo & - & - & + & - & - & + & - & - & - \\
\hline Trabajo en gran grupo (debate) & - & - & + & - & - & + & - & + & + \\
\hline
\end{tabular}




\section{Zeus Plasencia Carballo y Natalia Pérez Santana}

Análisis sobre la influencia de las estrategias orales previas al desarrollo de actividades de comprensión auditiva

\section{Estrategias orales llevadas a cabo durante las distintas fases de listening: +} Uso de la estrategia oral / - Abstención de la estrategia oral

Como se puede observar en la tabla, únicamente durante el desarrollo del pre-listening del audio A, se hace uso de la ayuda audiovisual; en este caso, vídeos de Youtube relacionados con los tipos de bailes que trataba el audio. Pudimos observar que esta clase de asistencia visual y auditiva despertó el interés del alumnado y le proporcionó más información respecto a los bailes en cuestión: i.e. los "capoeristas" son bailarines más atléticos y acrobáticos. Por otro lado, independientemente de la ayuda audiovisual, las estrategias orales propuestas se reducen al diálogo del profesor con algún alumno concreto, recayendo casi todo el peso del ejercicio en el propio profesor, quien ha de despertar expectativas, formular preguntas, orientar el interés y la dirección del ejercicio constantemente y; a través de cada uno de los pasos (pre-listening, listening y post-listening). Con ello, observamos que la autonomía del alumno queda bastante reducida a ciertos aspectos, como la inferencia y la creación de expectativas e hipótesis, y siempre de forma individual.

De igual manera, en el audio B podemos comprobar que se sigue un patrón similar al del primero, salvando el post-listening, paso en el que se abre un debate conjunto con toda la clase como participante. Esta estrategia oral es bastante positiva en dos aspectos: i) integra a todo el grupo y ii) se intercambian opiniones y puntos de vista entre los miembros del grupo. En este caso tampoco se promueve la autonomía del alumnado, salvo en el debate final al que ya hemos hecho referencia.

En el audio C, observamos que existe una mayor compensación en todos los aspectos, a excepción de la ayuda audiovisual. Desde el primer momento, se intenta que el alumnado sea capaz de llevar a cabo el proceso de comprensión por sí solo, aunque siempre acompañado por la orientación que pueda aportar el profesor. En este caso, se da especial atención al trabajo cooperativo, uno de los principios constructivistas que valora las relaciones interpersonales como fuente de conocimientos ${ }^{4}$. Por otro lado, aunque el profesor sigue siendo clave durante el desarrollo de la sesión, pasa a un segundo plano, tratando de supervisar y guiar la sesión hasta el objetivo marcado.

Un aspecto bastante interesante tras las sesiones de comprensión auditiva es la adaptación. Es decir, cuando los alumnos se acostumbran a la rutina de "hablar para entender mejor", observamos una mayor facilidad en la realización de las comprensiones auditivas, llegando incluso a producirse una disminución de las escuchas: de cuatro escuchas en el audio A, a tres en el audio C. Por otro lado, si estas estrategias orales promueven la autonomía y el trabajo cooperativo entre los alumnos a la hora

4 Vygotsky defendió que las relaciones interpersonales cumplen una función educativa de primer orden. 


\section{Zeus Plasencia Carballo y Natalia Pérez Santana}

Análisis sobre la influencia de las estrategias orales previas al desarrollo de actividades de comprensión auditiva

de entender los audios, también es cierto que las mismas estrategias ayudan al desarrollo del speaking y de la spoken interaction, dos de las destrezas más importantes durante el aprendizaje de una lengua, y de las más difíciles de promover dentro del aula. En la misma línea, podemos incluir la destreza del reading, debido a que en los audios A y B se acompañó el último con la lectura de la transcripción del texto oral.

Durante el desarrollo de nuestra experiencia, hemos podido llevar a cabo distintas estrategias o técnicas con el fin de activar los conocimientos previos del alumnado relacionados con la grabación que se iba a ejecutar. En el siguiente gráfico se facilita un esquema resumen sobre las tres audiciones llevadas a cabo en el grupo de $2^{\circ}$ de la ESO y las estrategias orales empleadas en las mismas, graduadas de 0 a 5 , atendiendo a su empleo en cada audio.

De la presente gráfica, entendemos que el alumnado recibe de buen grado todo tipo de apoyo audiovisual que sea capaz de despertar el interés sobre el tema en cuestión que se vaya a trabajar, además de activar los conocimientos que ya poseen para poder construir nuevos significados durante la comprensión auditiva.

Por otro lado, disponemos también, de la siguiente gráfica sobre estrategias orales empleadas en el transcurso de nuestra experiencia, indicando el grado de aceptación obtenido por parte del alumnado respecto a las mismas:

Independientemente del ya mencionado apoyo audiovisual, el grupo ha recibido con agrado la autonomía que confiere el diálogo alumno-alumno y el debate final que se solía hacer durante el post-listening. Los propios alumnos se ven capacitados para deducir, inferir y proponer hipótesis sobre lo que van a escuchar. Entre ellos mismos existe un espíritu cooperativo, poco explotado -dicho sea de paso-, que promueve el equilibrio dentro del grupo, es decir, lo que no entiende uno, lo puede explicar otro, y viceversa.

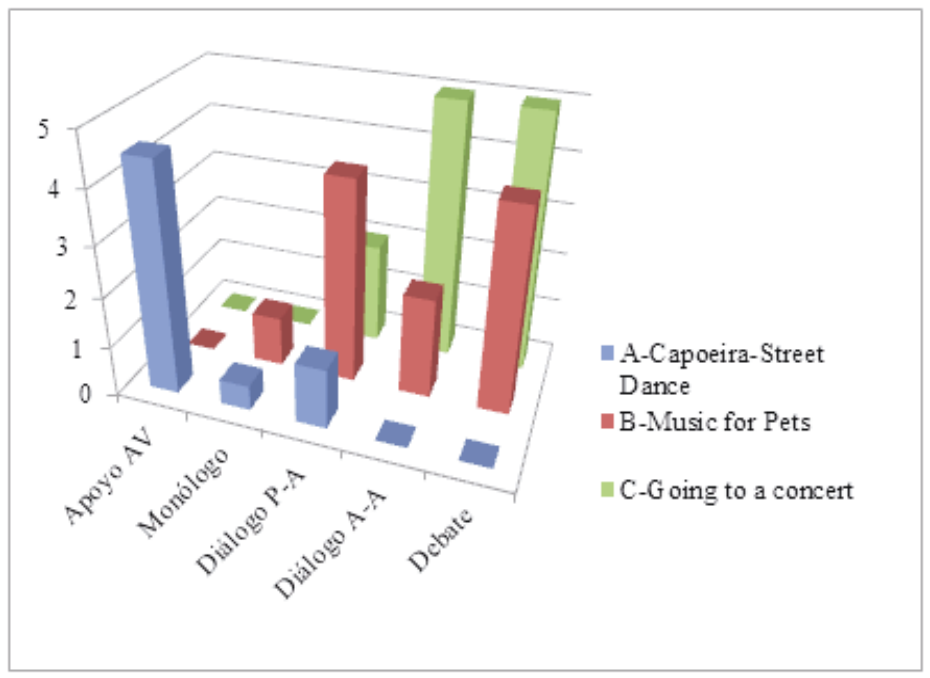

El Guiniguada, 23 (2014) • pp. 87-99 • eISSN: 2386-3374 


\section{Zeus Plasencia Carballo y Natalia Pérez Santana}

Análisis sobre la influencia de las estrategias orales previas al desarrollo de actividades de comprensión auditiva

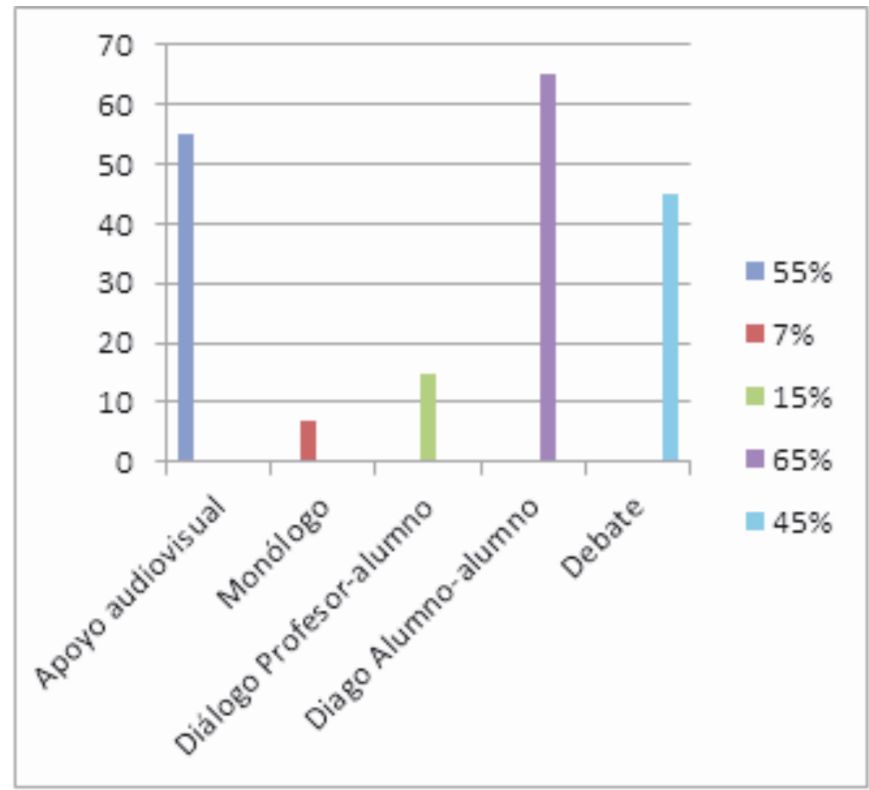

Del mismo modo, y aunque los alumnos disfruten de la libertad de poder discutir sobre el listening entre ellos, no desestiman la necesidad de que el profesor actúe como guía durante la sesión y pueda orientarles en la dirección correcta a la hora de realizar un ejercicio de comprensión auditiva. Sin embargo, e incidiendo una vez más en la figura del profesor, el alumnado sí que rechaza el monólogo que tradicionalmente realiza el profesor en este tipo de sesiones, limitando así su participación activa.

\section{Discusión}

Teniendo en cuenta todo lo anterior, podemos afirmar que las estrategias orales empleadas durante las sesiones de $2^{\circ}$ de ESO sí que facilitaron la comprensión de textos orales. Tanto el apoyo audiovisual como la interacción oral entre alumnos y profesor han contribuido a una mejor preparación y activación de los conocimientos previos del alumno en cuanto a la comprensión auditiva, como acertadamente argumentan Rost (2002) y Flowerdew \& Miller (2005). Son, pues, más que obvias las ventajas que aporta el empleo de las actividades orales desarrolladas con el grupo y evidenciamos, una vez más, las pocas garantías que ofrece a largo plazo el modelo tradicional (dos escuchas y ejercicios mecánicos sobre el audio) a la hora de enfocar este tipo de ejercicios. Hemos tratado de partir de una base constructivista en la que el profesor facilite, oriente y ayude al alumno durante el proceso de aprendizaje. Dichas actividades han 


\section{Zeus Plasencia Carballo y Natalia Pérez Santana}

Análisis sobre la influencia de las estrategias orales previas al desarrollo de actividades de comprensión auditiva

reportado resultados positivos en cuanto a la comprensión auditiva dentro del aula, además de impulsar el desarrollo de otras destrezas como el speaking y spoken interaction. En otras palabras, el uso de estas estrategias orales ha promovido que distintos aspectos de la lengua extranjera se ejerciten al mismo tiempo, llevándose a cabo un uso más completo, intensivo y natural de la misma.

\section{BibliografíA}

Chang, S. (2012). Approaching L2 Listening Comprehension for Advanced Learners: Using Reading as a PreListening Task, EE.UU: Penn State University Press.

Coll, C. (1990). Aprendizaje escolar y construcción del conocimiento, Barcelona: Paidós Educador.

Coll, C. et al (1993). El constructivismo en el aula, Barcelona: Graó.

Coll, C. et al (2010). Desarrollo, aprendizaje y enseñanza en la educación Secundaria, Barcelona: Graó.

Consejería de Educación, Cultura y Deporte (2007). Decreto 126/2007, de 24 de mayo, por el que se ordena $y$ desarrolla el Currículo de la Educación Secundaria Obligatoria en Canarias, $\mathrm{BOC} \mathrm{n}^{\circ} 113$, jueves 7 de junio 2007, Gobierno de Canarias.

Consejería de Educación, Cultura y Deporte (2008). Decreto 202/2008, de 30 de septiembre, por el que se ordena y desarrolla el Currículo de Bachillerato en Canarias, BOC $\mathrm{n}^{\circ}$ 204, viernes 10 de octubre 2008, Gobierno de Canarias.

Flowerdew, J. y Miller, L. (2005). Second Language Listening: Theory and Practice. Cambridge, UK: Cambridge University Press.

Instituto Canario de Evaluación y Calidad Educativa (ICEC). Pruebas de Diagnóstico 2011-2012. Febrero 2013, Gobierno de Canarias.

Instituto Cervantes (2001). Marco Común Europeo de Referencia para las Lenguas: aprendizaje, enseñanza, evaluación, Consejo de Europa. Madrid: Instituto Cervantes.

Ministerio de Educación, Cultura y Deporte (2004). Guía didáctica del Portfolio europeo de las lenguas para la enseñanza Secundaria. Madrid: Omagraf, SL.

Ministerio de Educación, Cultura y Deporte (2006). Ley Orgánica 2/2006 de Educación, de 3 de mayo por la que se ordena el Currículo de las enseñanzas no universitarias del sistema educativo español. $\mathrm{BOE} \mathrm{n}^{\circ} 106$, jueves 4 mayo 2006. Gobierno de España.

Piaget, J. (1999). La psicología de la inteligencia, Barcelona: Editorial Crítica.

Rost, M. (2002). Teaching and Researching Listening. Essex, UK: Pearson Education Limited.

zeuspc1388@gmail.com 